\title{
Biochemical and Textural Ripeness Assessment of Avocado Fruit from Different Origins
}

Sandra Landahl, Marjolaine D. Meyer and Leon A. Terry*

Plant Science Laboratory, Cranfield University, Bedfordshire, MK43 0AL, UK

* E-mail address: 1.a.terry@ cranfield.ac.uk

Keywords: Persea americana 'Hass', carbohydrates, fatty acids, stress-relaxation

\begin{abstract}
Stage of ripeness and quality of avocado is notoriously difficult to assess by conventional methods. Texture is a very important determinant of avocado fruit quality and can change radically during storage. The difficulties in determining avocado quality are related, in part, to the spatial heterogeneity of fruit characteristics during ripening. The aim of this study was to assess the discriminatory capabilities of physiochemical properties to determine ripeness of imported 'Hass' avocado fruit. Fruit were stored at $12^{\circ} \mathrm{C}$ and sampled at regular intervals. Using a previously unreported method, the texture of different slices from individual fruit was measured during ripening. Maximum load, elasticity and viscosity of fruit tissue was measured using an Instron 5542 universal testing machine fitted with a $500 \mathrm{~N}$ or $5 \mathrm{~N}$ load cell. The same tissue slice was then further processed prior to subsequent quantification of non-structural carbohydrates (NSCs) and fatty acid methyl esters using standard high performance liquid chromatography coupled to evaporative light scattering detection and gas chromatography coupled to flame ionisation detection, respectively. Spatio-temporal differences in maximum load, elasticity, viscosity, NSCs and fatty acid composition were found in avocados from different origins. Results of measured texture and target analytes were used to differentiate avocado fruit into definable groups using partial least squares discriminant analysis.
\end{abstract}

\section{INTRODUCTION}

Avocado (Persea americana Mill.) fruit are an attractive addition to the diet due to their unique flavour and texture. In addition, avocados have reported health benefits (Ashton et al., 2006; Ding et al., 2007; Henrotin, 2008), e. g. research has shown that eating avocado fruit flesh may contribute to lowering cholesterol levels (Ledesma et al., 1996). Avocado mesocarp tissue has high concentrations of desired nutrients such as unsaturated fatty acids and seven carbon carbohydrates.

The OECD (2004) recommends to rate the quality of avocado fruit according to size, estimated oil content (dry matter), absence of defects and fruit firmness. The degree of ripeness of many climacteric fruits like avocado can be correlated with fruit firmness. It has been shown that fruit often do not soften uniformly due to physiological gradients. Spatial changes in dry matter and pigments have been investigated during avocado ripening (Schroeder, 1985; Ashton et al., 2006). However, the distribution of nonstructural carbohydrates (NSCs) and fatty acids have been not examined. Therefore, in this research the fatty acid composition, NSC content as well as texture of avocado fruit were determined in a number of predefined localities within individual fruit during ripening and analysed using chemometric analysis. A known test procedure for measuring viscoelastic properties of fruit tissues (Sakurai and Nevins, 1992) was optimized in order 
to minimize tissue damage, which allows biochemical analyses on the same avocado mesocarp tissue. This textural analysis was performed by means of a stress-relaxation test.

The present study aimed at enhancing spatio-temporal understanding of the ripening process in 'Hass' avocado fruit sourced from three different countries (viz. Spain, Peru, Chile), which are major suppliers to the UK and thus may ultimately assist quality selection in the import-country by aiding prediction of ripeness.

\section{MATERIALS AND METHODS}

\section{Reagents}

Methyl palmitate, methyl palmitoleate, methyl oleate, methyl linoleate, methyl linolenate, sucrose, D-glucose, D-fructose and D-mannoheptulose standards were purchased from Sigma (Dorset, UK). Perseitol (D-glycero-D-galacto-heptitol) was obtained from Industrial Research Ltd. (IRL - Fine Chemicals, New Zealand). All other chemicals used were of analytical grade and purchased from Fisher Scientific Chemicals (Leics., UK).

\section{Plant material}

All imported avocado fruit (size code 16 fruit; OECD, 2004) were supplied by M. W. Mack Ltd. (Kent, UK). Three experiments were conducted on fruit not commercially ripened and originating from Malaga, Spain (late season 2007), La Libertad, Peru (early season 2007) and Quillota Province, Chile (mid season 2007). The physiological age of the imported avocados was different at arrival due to the respective distance from country of origin to the UK. Fruit were not treated with 1-methylcyclopropene. Produce was held in the laboratory overnight at $5-6^{\circ} \mathrm{C}$, after which fruit were placed into hermetically sealed polypropylene boxes (app. $32 \mathrm{~cm} \times 14.5 \mathrm{~cm}$ x $28 \mathrm{~cm}$ ). Controlled ripening was initiated using exogenous ethylene $\left(100 \mu \mathrm{L} \mathrm{L}^{-1}\right.$; BOC Gases Ltd., Surrey, UK; Terry et al., 2007b) and avocado fruit kept overnight at ambient temperature. After $24 \mathrm{~h}$ the boxes containing the avocado fruit were opened and transferred to $12^{\circ} \mathrm{C}$ for subsequent ripening. Fruit subsamples (Spanish $n=3$, Peruvian and Chilean $n=6$ ) were randomly selected four times at regular intervals.

\section{Sample preparation}

Each avocado fruit was cut vertically using a sharp knife, with the stem facing the operator. The left half was placed with the flat planar surface downwards and a $1 \mathrm{~cm}$ thick slice cut horizontally from the stem end; this piece was discarded. Thereafter, ten slices were sequentially cut starting from the apical end (near stem) towards the basal end (rounded) of the fruit. The slices number 1, 5 and 9 were selected resulting in three ca. $1 \mathrm{~cm}$-thick slices for textural measurement, which took place immediately after cutting. The seed (if appropriate) and skin were removed prior to texture tests.

\section{Textural evaluation}

All texture tests were performed on an Instron (model 5542, MA) uni-axial testing machine equipped with calibrated $500 \mathrm{~N}$ or $5 \mathrm{~N}$ load cell, depending on the maximum loads recorded on the test day. Textural tests for each slice were done vertically such that the planar surface of the slice was in contact with the sample holder. The machine was programmed (Bluehill 2, version 2.11, Instron) such that the probe indented the sample to

a depth of $0.6 \mathrm{~mm}$ with the cross head speed set at $10 \mathrm{~mm} \mathrm{~min}^{-1}$ and then held this 
position for $1 \mathrm{~min}$ (Sakurai and Nevins, 1992). A right circular cylinder probe of $6 \mathrm{~mm}$ diameter was used; the low indentation depth ensured minimal destruction of the tissue sample. The mean value of three replicate measurements was calculated for each slice. Force $[\mathrm{N}]$, deformation $[\mathrm{mm}]$ and time [s] were recorded as raw data throughout each test using the Bluehill software (at force changes of $20 \mathrm{mN}$ or $2 \mathrm{mN}$ depending on the load cell used, or at a maximum rate of $10 \mathrm{~s}^{-1}$ ). The apparent elasticity modulus was calculated from the highest slope of the first portion of the stress-relaxation curve. This slope was found by least square fit on six regions of the curve. Minimum relaxation time, was calculated using Matlab software (7.0.4.365 R14 Service Pack 2, The MathWorks Inc., MA). The exponential law of relaxation of the Maxwell-model was applied to find the relaxation time of the second part of the stress-relaxation curves recorded for every avocado slice. The minimum relaxation time values were calculated.

At least $5 \mathrm{~g}$ of three measured mesocarp tissue of slices (apex, middle, base) were cut into small pieces and immediately snap-frozen in liquid nitrogen after testing and stored at $-40^{\circ} \mathrm{C}$ to preserve them prior to biochemical analysis $(\mathrm{n}=180)$.

\section{Fatty acids and NSCs extraction and quantification}

Frozen mesocarp tissue samples of the slices $(n=180)$ were freeze-dried in a Christ freeze dryer with cooling trap ALPHA (100 400, Osterode, Germany) for 7 days at $0.05 \mathrm{hPa}$ (Terry et al. 2007a). Dry mass (DM) was recorded and samples returned to $40^{\circ} \mathrm{C}$ until further processing. Lyophilized mesocarp tissue was ground, weighed (to $1 \mathrm{~g}$ ) and a hexane extraction performed as described by Meyer and Terry (2008). The recovered oil and powder were weighed and returned to $-40^{\circ} \mathrm{C}$ for subsequent fatty acid and NSC analysis, respectively.

Fatty acid methyl esters (FAMEs) were produced according to International Olive Oil Council (IOOC, 2001) modified by Meyer and Terry (2008). The fatty acid composition was calculated as percentage of total of the five detected FAMEs, after comparison of peak areas of samples and peak areas of mix of standards of known composition.

NSCs were extracted and quantified as previously described (Terry et al., 2007a) with some modification according to (Meyer and Terry, 2008). Starch was not analysed. Mixed standards of known composition and concentration were used and presence of each NSC quantified by comparing sample peak areas to the standards. The minimum detection limit for the NSCs was $20 \mathrm{mg} \mathrm{g}^{-1}$ powder residue. Results below detection limit were set at zero, which causes an unavoidable underestimation of the mean value of NSC

content. For clarity, the number of values below detection are reported for particular regions within the avocado fruits.

\section{Chemometric analysis}

All statistical analyses were carried out using a trial version of The Unscrambler (9.7, Camo Software Inc., NJ). Multivariate analysis was performed using partial least squares discriminant analysis (PLS-DA). Data were centred and variables were weighted by dividing with the standard deviation. A full cross validation was done to perform a Martens' uncertainty test to identify the important variables in the prediction of origin, storage duration and the vertical location of the tissue slice within the fruit (y-variables or "treatment"). Then the PLS-DA was repeated for each y-variable with the important variables only. Total explained variance, loading plots and score plots were used for interpretation. 
Different test days were combined by storage duration and termed: "before" = day 0 , "early" = day 3 or 4 , "later" = day 6 or 7 , "store end" = day 10 or 11 . The x-variables included in the PLS-DA were the measured parameters. All avocado tissue samples, which were tested for fatty acids and NSCs, were included in the analysis $(n=180)$.

\section{RESULTS AND DISCUSSION}

\section{Country of origin}

The scores of fruit from Peru were clearly separated in the first latent variable (LV, Fig. 1). The scores of fruit from Chile and Spain formed groups but overlapped according to LV2. The variability due to origin of the fruit was 53\% explained by LV1 and $17 \%$ by LV2. The most important variables in LV1 were oil content, oleic, palmitic, palmitoleic and linoleic acid. The most important variable in LV2 was mannoheptulose.

The score plots illustrate a significant difference between avocados from Peru and the other two origins. That said, the fatty acid composition measured during the tests is in the range of that reported by others (Eaks, 1990; Requejo-Tapia et al., 1999; Ozdemir and Topuz, 2004; Vekiari et al., 2004). The distinct oil content and fatty acid composition found in fruit from three countries of origin could be related to seasonal variability (Requejo-Tapia et al., 1999) and/or to different biological age.

\section{Storage time}

The scores of fruit measured before storage, during later ripening and at the end of storage were grouped according to LV1 (Fig. 2). Scores of fruit of the later ripening stage overlapped with scores of avocados at the end of storage but not with fruit tested before storage. The variability was explained $(29 \%)$ by LV1. Textural parameters were the most important variables able to predict effect of different storage times. Thus, the stressrelaxation parameters were well suited to describe avocado fruit ripening.

\section{Vertical location within fruit}

The classification of tissue from different vertical locations within the avocado fruit was not clear when all origins and storage times were included in the PLS-DA, since the explained variance of location within the fruit amounted to only $21 \%$ for the first two LVs. That said, a trend was found which distinguished the apical tissue slices from the base (data not shown). The most important variables were linoleic and linolenic acid, and mannoheptulose. Heterogeneity within the fruit was highlighted by the count of values of NSC content below detection (Table 1).

\section{CONCLUSIONS}

Avocado fruit were sourced from three different producing countries, which are major suppliers for import into the UK. The spatio-temporal ripening process of 'Hass' avocado fruit was analysed by means of PLS-DA. The heterogeneity of textural properties and nutritional parameters within a fruit was described. The examination of not only physical development, but also biochemical changes of the same samples provided a greater insight into the ripening process of the mesocarp tissue. Spatial distribution within avocado fruit from the same consignment showed that sampling location is critical when interpreting fruit status and ultimately quality. The current study has demonstrated that stress-relaxation measurements are well suited to monitor origin and ripeness of avocado fruit. 


\section{ACKNOWLEDGEMENTS}

This work forms part of a larger FoodLink project (AFM 235) and is financially supported by the UK Government (Department for Environment, Food and Rural Affairs, Defra) and UK industry representatives (Lambda Photometrics Ltd., M. W. Mack Ltd., Marks and Spencer plc, Unilever R\&D). The support from Prof. George Jeronimidis, University of Reading, is greatly appreciated. The technical help of Balwinder Cook, Rosemary Burns and Allen Hilton, Cranfield University, is gratefully acknowledged. Dr. Conrad Bessant, Cranfield University, is thanked for assistance with chemometric analysis.

\section{Literature cited}

Ashton, O.B.O., Wong, M., McGhie, T.K., Vather, R., Wang, Y., Requejo-Jackman, C., Ramankutty, P. and Woolf, A.B. 2006. Pigments in Avocado Tissue Oil. J. Agric. Food Chem. 54:10151-10158.

Ding, H., Chin, Y.-W., Kinghorn, A.D. and D'Ambrosio, S.M. 2007. Chemopreventive characteristics of avocado fruit. Seminars in Cancer Biol. 17:386-394.

Eaks, I.L. 1990. Change in the fatty acid composition of avocado fruit during ontogeny, cold storage and ripening. Acta Hort. 269:141-152.

Henrotin, Y. 2008. Avocado/soybean unsaponifiable (ASU) to treat osteoarthritis: a clarification. Osteoarthritis and Cartilage. 16:1118-1119.

IOOC (International Olive Oil Council). 2001. COI/T20/Doc. No. 24.

Ledesma, R.L., Frati Munari, A.C., Hernandez Dominguez, B.C., Cervantes Montalvo, S., Hernandez Luna, M.H., Juarez, C. and Moran Lira, S. 1996. Monounsaturated fatty acid (avocado) rich diet for mild hypercholesterolemia. Arch. Med. Res. 27:519-523.

Meyer, M.D. and Terry, L.A. 2008. Development of a rapid method for the sequential extraction and subsequent quantification of fatty acids and sugars from avocado mesocarp tissue. J. Agric. Food Chem. 56:7439-7445.

OECD. 2004. Avocados. International Standardisation of Fruit and Vegetables. 8-31.

Ozdemir, F. and Topuz, A. 2004. Changes in dry matter, oil content and fatty acid composition of avocado during harvesting time and post-harvesting ripening period. Food Chem. 86:79-83.

Requejo-Tapia, L.C., Woolf, A.B., Rougham, G., Schroeder, R., Young, H. and White, A. 1999. Avocado postharvest research: 1998/99: Seasonal changes in lipid content and fatty acid composition of 'Hass' avocados. Rep. to the NZ Avocado Industry Council. HortResearch Client Report 2000/1.

Sakurai, N. and Nevins, D.J. 1992. Evaluation of Stress-Relaxation in Fruit Tissue. HortTechnol. 2:398-402.

Schroeder, C. A. 1985. Physiological gradient in Avocado fruit. California Avocado Society Yearb. 69:137-144.

Terry, L.A., Chope, G.A., Giné Bordonaba, J. 2007a. Effect of water deficit irrigation and inoculation with Botrytis cinerea on strawberry (Fragaria $\mathrm{x}$ ananassa) fruit quality. J. Agric. Food Chem. 55:10812-10819.

Terry, L.A., Ilkenhans, T., Poulston, S., Rowsell, L. and Smith, A.W.J. 2007b. Development of new palladium-promoted ethylene scavenger. Posth. Biol. Technol. 45:214-220.

Vekiari, S.A., Papadopoulou, P.P., Lionakis, S. and Krystallis, A. 2004. Variation in the composition of Cretan avocado cultivars during ripening. J. Sc. Food Agri. 84:485492. 


\section{Tables}

Table 1 Count of values below detection limit for non-structural carbohydrates from different regions within 60 avocado fruit.

\begin{tabular}{lccc}
\hline Slice & Sucrose & Mannoheptulose & Perseitol \\
stem-end & 20 & $\mathbf{9}$ & $\mathbf{8}$ \\
middle & 15 & 22 & 12 \\
base & $\mathbf{1 2}$ & 31 & $\mathbf{5}$ \\
\hline
\end{tabular}

\section{Figures}

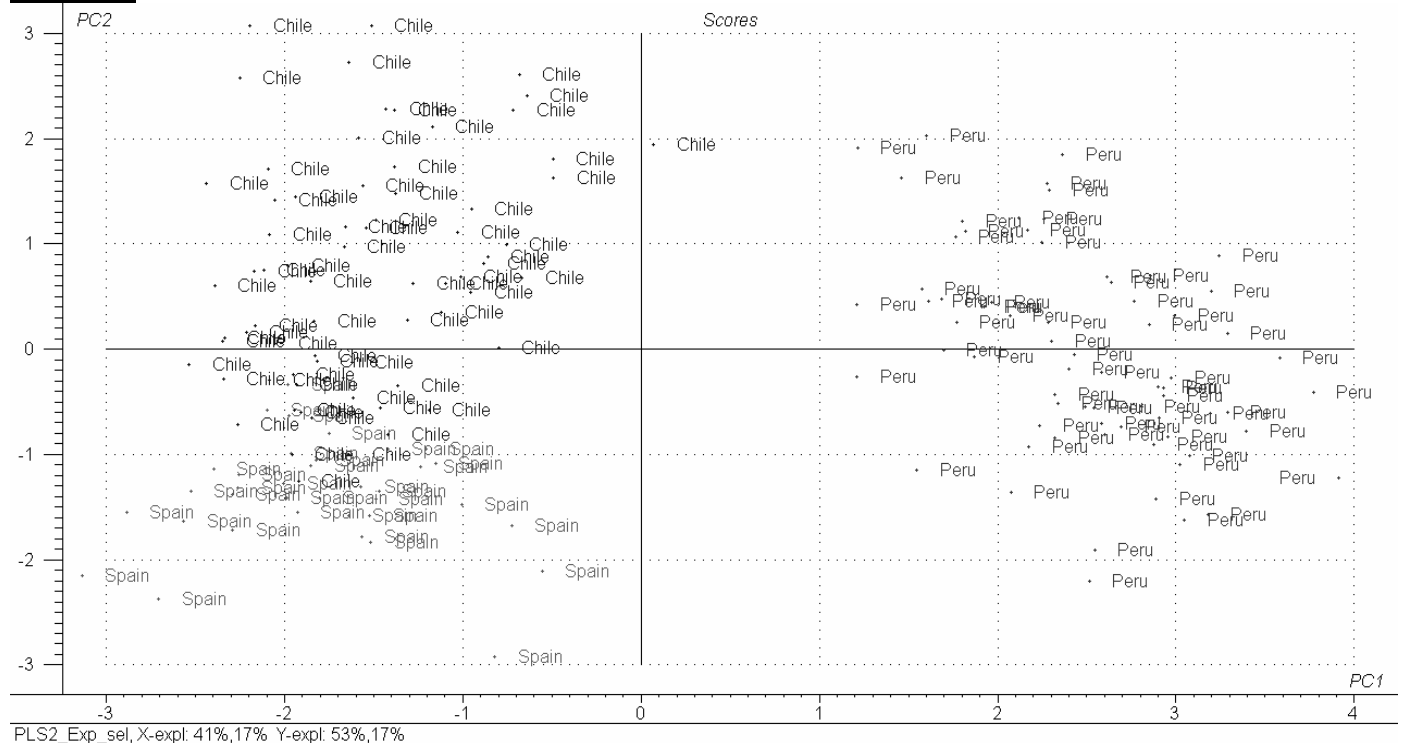

Figure 1 Score plot of PLS-DA to predict three measured batches from different countries of origin (60 avocado fruit).

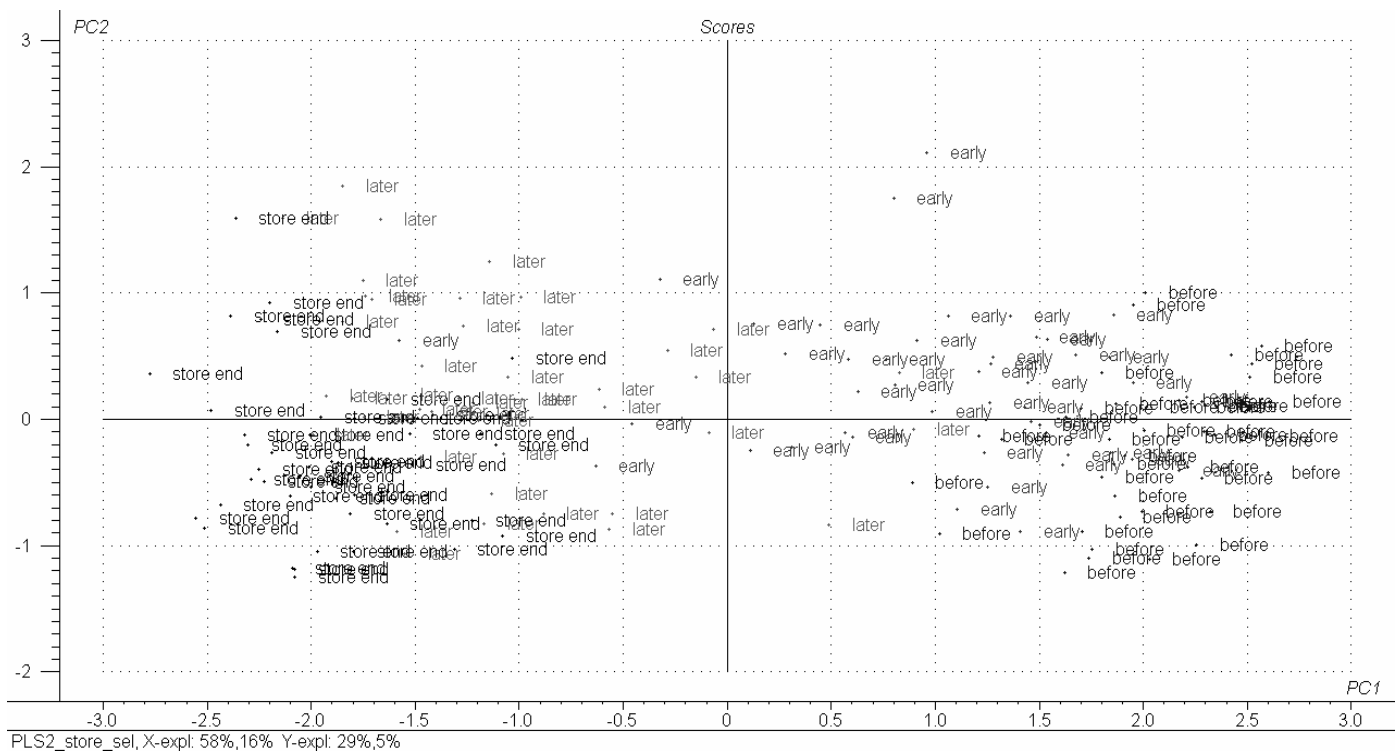

Figure 2 Score plot of PLS-DA to predict four measured storage times of 60 avocado fruit (samples $\mathrm{n}=180)$. 